\title{
Hamlet de Talma à Mounet-Sully : « une ressemblance immortelle »
}

Isabelle Schwartz-Gastine

\section{OpenEdition}

Édition électronique

URL : http://journals.openedition.org/shakespeare/3906

DOI : 10.4000/shakespeare.3906

ISSN : 2271-6424

Éditeur

Société Française Shakespeare

Référence électronique

Isabelle Schwartz-Gastine, "Hamlet de Talma à Mounet-Sully : « une ressemblance immortelle » », Actes des congrès de la Société française Shakespeare [En ligne], 35 | 2017, mis en ligne le 11 mai 2017, consulté le 02 mai 2019. URL : http://journals.openedition.org/shakespeare/3906 ; DOI : 10.4000/ shakespeare.3906

Ce document a été généré automatiquement le 2 mai 2019.

(c) SFS 


\title{
Hamlet de Talma à Mounet-Sully : « une ressemblance immortelle»
}

\author{
Isabelle Schwartz-Gastine
}

\section{Le texte initial de Ducis : La tragédie d'Hamlet}

\section{Interprétation de Molé}

1 Tandis qu'en 1769 David Garrick organisait à Stratford le Jubilé dédié à Shakespeare ${ }^{1}$, Jean-François Ducis présentait sa tragédie devant le Comité de Lecture de la ComédieFrançaise. Ne parlant pas anglais², Ducis s'était largement inspiré de la version de PierreAntoine de La Place dont les huit volumes de son édition du Théâtre Anglois avaient paru entre 1746 et $1749^{3}$. La Place, qui, lui, maitrisait l'anglais puisqu'il avait fréquenté le Collège des Jésuites Anglais de Saint-Omer entre 1714 et $1721^{4}$, s'était inspiré de l'édition des œuvres de Shakespeare qu'Alexander Pope fit paraître entre 1723 et 1725, bientôt suivie d'une deuxième édition augmentée, en $1728^{5}$. Selon John Golder, La Place lui-même aurait suggéré à Ducis de continuer l'œuvre qu'il avait commencée et surtout, de mener le théâtre shakespearien jusque sur la scène française ${ }^{6}$.

Ducis avait commencé à travailler sur sa pièce en 1767, deux ans après le long séjour à Paris de Garrick ${ }^{7}$. Parlant français de par ses ascendances bordelaises ${ }^{8}$, Garrick avait été l'hôte de tous les Salons littéraires parisiens dans lesquels il avait amplement montré son art théâtral et son enthousiasme pour Shakespeare, et ensuite, il avait entretenu une correspondance suivie avec des personnalités du monde artistique et littéraire. Dans une lettre, Ducis lui précisait que pour satisfaire au goût français, il était dans l'obligation d'éliminer les «irrégularités sauvages dont la pièce abonde " ${ }^{9}$. Cependant, malgré ses tentatives pour calquer son œuvre sur le style de Corneille ou de Racine, il essuya un refus devant le Comité de Lecture du Théâtre-Français le 20 mai 1768, alors que sa première pièce avait été acceptée et jouée quelques mois plus tôt ${ }^{10}$. Ce n'est que le 17 juillet 1769 , après plusieurs autres tentatives et assorti d'une demande de modifications substantielles, que le Comité accepta le manuscrit ${ }^{11}$. Ce cheminement tortueux montre le peu d'empressement du Comité de Lecture composé des principaux sociétaires pour faire 
jouer une pièce d'inspiration shakespearienne sur la scène du premier théâtre royal français.

3 Comme il fallait combler une défection, la pièce fut programmée immédiatement, et donc, Ducis procéda en toute hâte à la distribution des rôles, puisque, selon la coutume du Théâtre-Français, c'était à l'auteur que revenait cette tâche. Il se heurta au refus de Lekain, le premier acteur tragique que Voltaire admirait tant, qui prétexta des innovations « barbares » inacceptables ${ }^{12}$. Ducis se tourna alors vers l'acteur qui avait lu sa pièce au Comité, François Molé, choix hardi car Molé était chef d'emploi du répertoire comique $^{13}$. La pièce fut créée dans l'après-midi du samedi 30 septembre 1769 dans la vaste Salle du Jeu de Paume de l'Etoile, rue des Fossés Saint-Germain des Prés, alors en bien triste état ${ }^{14}$. L'assemblée, composée de presque mille spectateurs, assura le succès de la pièce par de confortables bénéfices ${ }^{15}$. A l'issue de la représentation "le parterre " demanda l'auteur, faveur parcimonieusement accordée et donc, signe tangible de contentement. Etant donné ce succès, fait exceptionnel, la pièce fut représentée encore quatre fois, début octobre ${ }^{16}$.

Néanmoins, des voix discordantes se firent entendre. Bachaumont, voltairien convaincu, s'insurgea contre la façon dont Ducis avait imité Shakespeare :

Quelques-uns, comme M. de Voltaire, s'étaient contentés d'en prendre les beautés de détail et de les transposer dans leurs pièces. M. Ducis s'est approprié cette carcasse et en a formé un drame régulier, mais qui, dénué de ces endroits neufs et terribles, dont on s'était emparé avant lui, n'a plus été qu'une tragédie ordinaire ${ }^{17}$.

Diderot affirma qu'il «s'accommoderai[t] encore mieux du monstre de Shakespeare que de l'épouvantail de M. Ducis ${ }^{18} »$. Alors, Ducis se remit à l'ouvrage, si bien que pour la deuxième représentation, quatre jours plus tard (mercredi 4 octobre 1769), le texte porté à la scène avait subi des transformations considérables.

6 Dans sa lettre écrite à Garrick tandis qu'il composait sa pièce, Ducis « [regrettait] de ne pouvoir y transposer l'ombre terrible qui expose le crime et demande vengeance ${ }^{19}$. Vingt ans auparavant, en 1749, Voltaire avait été critiqué pour avoir introduit un spectre dans Sémiramis ${ }^{20}$. Cette question redevenait d'actualité. Pour les plus conservateurs, comme Charles Collé, le principe de bienséance interdisait de mentionner les êtres venus du royaume des ténèbres dans une tragédie et de les représenter sur la scène du premier théâtre royal ${ }^{21}$. Pour L'année Littéraire, en revanche, le spectre était indispensable :

L'apparition du fantôme est inhérente au sujet, elle est consacrée ; sans elle toutes les convulsions du jeune prince ne touchent plus et ne sont plus regardées par les spectateurs sensés que comme les rêves d'un cerveau malade, auxquels cependant il sacrifie la nature, l'amour et lui-même ${ }^{22}$.

7 C'était aussi l'opinion de Diderot qui considérait que l'absence de fantôme était l'une des faiblesses de cette tragédie. Il précisait cependant que, dans la pièce de Shakespeare, le fantôme était «juste » en demandant à Hamlet d'épargner sa mère puisqu'elle était repentante, contrairement à celui de Ducis qui l'obligeait à la frapper ${ }^{23}$.

8 À la première, le 30 septembre 1769, "L'Ombre " n'était pas visible, mais le spectre s'incarnait en une voix vengeresse qui s'adressait à Hamlet, alors qu'il était avec sa mère (scène IV, page 984): «Frappe!». Sans indication précise, on peut supposer que Delaporte, le secrétaire-souffleur, prenait en charge cette réplique. À la deuxième représentation, quatre jours plus tard, cette réplique fut supprimée de la main même de Ducis, mais la violence rétributive du fils fut conservée. Après la didascalie Il tire son poignard, Hamlet réplique : «J'entends sa voix. / Oui, frappons », suivi de l'indication : Sa 
mère tombe à ses pieds, il se retourne pour la frapper. Sans la réplique dramatique du spectre, il revenait à l'acteur d'imprimer sur son visage et dans son corps l'horreur de cette injonction terrible. Ce fut l'un des grands moments dramatiques de la mise en scène car Molé produisait un effet saisissant. Il usait de pantomime, inspirée de la commedia dell'arte, qu'il pratiquait dans son registre comique et qui plaisait tant aux spectateurs du parterre. Il devait s'inspirer également de Garrick qui avait mimé Macbeth en proie à l'hallucination du poignard qui apparait devant ses yeux ${ }^{25}$. Il y eut certes des opinions discordantes, celle de Collé évidemment ; en revanche, Madame du Deffand, salonnière et amie de Voltaire, fut parmi les nombreuses dames qui se pâmaient d'extase tandis que Molé se lançait « à corps perdu dans les convulsions les plus effroyables de la pantomime $26 \gg$.

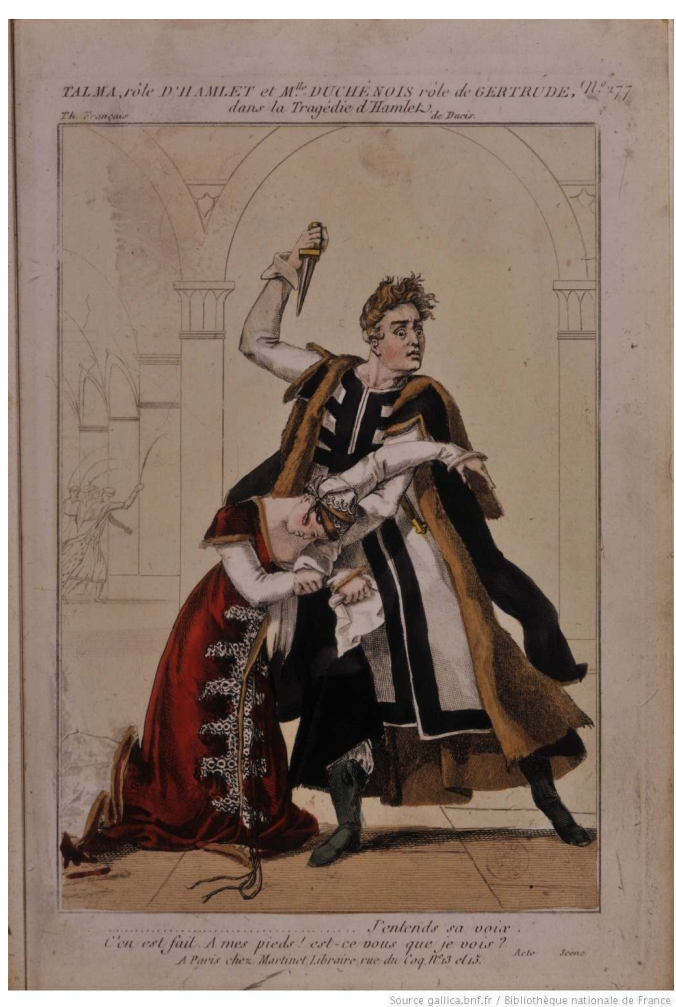

Illustration 1 : Talma et Mlle Duchesnois dans Hamlet, de Ducis, d'après Shakespeare, gravure non datée.

Source Gallica.bnf.fr / Bibliothèque nationale de France

Une modification, imaginée par Ducis pour se conformer aux règles classique tout en maintenant le ressort tragique venant de l'au-delà, connut un succès thématique et scénique unanime. Alors que La Place avait conservé la scène dans laquelle Hamlet parle théâtre avec les comédiens, Ducis l'avait éliminée sous prétexte que concevoir un Prince héritier de la couronne royale conversant avec de simples "acteurs de campagne" dépassait les limites de la bienséance ${ }^{27}$. Aussi, plutôt que de recourir à la pièce dans la pièce, impensable dans une perspective dramaturgique classique, Ducis mit en œuvre un autre stratagème pour susciter des aveux de la part de Gertrude. Juste avant le passage cité précédemment, Horatio, renommé Norceste, avait transmis à Hamlet une urne funéraire, qu'il venait de déterrer du cimetière, contenant les cendres du défunt Roi. Pour faire avouer sa mère, Hamlet lui pressait violemment l'urne contre son sein, puis celle-ci 
s'écroulait dans un fauteuil. Diderot trouva que ce moment de confrontation était « une très belle scène » bien plus émouvante que l'original shakespearien :

Vous avouerez que ces cendres confrontées à l'homicide sont un moyen de découvrir le crime, bien plus naturel, plus décisif et plus tragique que cette pièce que fait jouer Hamlet dans l'anglais pour pénétrer un mystère de cette importance. Claudius et la reine peuvent être indignés d'une représentation qui semble imaginée pour les accuser, et leur fuite n'annonce pas alors leur conviction ${ }^{28}$.

Sur une table, trônait l'urne, majestueuse et accusatrice, rappelant sans cesse la culpabilité de Gertrude. Ce moment, «le plus riche et celui qui a été le plus applaudi », encore selon Diderot, qui recueillit le suffrage du parterre (qui applaudit) aussi bien que des lettrés (qui le discutèrent), fut considéré comme une amélioration bénéfique de l'original ${ }^{29}$.

11 Le problème de cette tragédie était son dénouement. Jamais satisfait, à la recherche d'effets, Ducis reprit sans cesse son ouvrage, produisant une nouvelle version dès la reprise en décembre 1769 [Manuscrit 266]. Contrairement au héros shakespearien, Hamlet restait en vie et succédait à son père comme roi du Danemark. Nul Fortinbras pour prendre le pouvoir, en effet, il aurait été sacrilège de faire s'arrêter brusquement une lignée royale sur la scène du Théâtre-Français. Dans les versions les plus heureuses, Hamlet se mariait avec Ophélie, la fille de Claudius, après avoir reçu la bénédiction de Gertrude mourante. Dans d'autres versions, Ophélie, cornélienne, reniait Hamlet par fidélité à la mémoire de son père, et dans ce cas, pour Hamlet, la vie était pire que la mort, comme le fait sentir le distique conclusif dont le dernier mot, "mourir », termine la tragédie : «Mais je suis homme et Roi. Réservé pour souffrir, / Je saurai vivre encor; je fais plus que mourir ${ }^{30} »$.

Il y eut d'innombrables changements, dès la première publication de 1770 , et pour la quinzaine d'éditions successives qui suivirent. Mais la séquence du poignard et celle de l'urne furent conservées, devenant les moments emblématiques de cette tragédie et assurant un succès scénique sans partage à la Comédie-Française et bien au-delà des frontières de la France.

\section{Ducis-Talma}

13 Ce siècle avait trois ans lorsque le 4 avril 1803 (14 Germinal première décade de l'An XI de la République), François-Joseph Talma reprit le rôle d'Hamlet au Théâtre de la Porte Saint-Martin ${ }^{31}$ pour une représentation à bénéfice en faveur de Charles Labussière. Acteur de second ordre, Labussière avait officié comme « secrétaire enregistreur au bureau des pièces accusatrices » du Comité de Salut Public de Saint Just durant la Terreur en 1793, mais avait réussi à sauver plus de mille prisonniers dont certains de ses anciens camarades accusés de jouer une pièce contre-révolutionnaire dans leur Théâtre de la Nation $^{32}$. En 1791, le Théâtre-Français avait fait scission: le Théâtre de la Nation était resté fidèle au répertoire classique tandis que le Théâtre de la République, mené par Talma, regroupait ceux qui voulaient favoriser la création contemporaine. Les deux théâtres étant en concurrence, Talma avait été soupçonné, de par ses sympathies girondines, d'avoir influé sur l'accusation de ses anciens camarades ${ }^{33}$. La réunion des deux troupes fut décrétée en 1799 et tous, non sans heurts, réintégrèrent la rue de Richelieu. Entre temps, au Théâtre de la République, Talma était devenu le tragédien favori de Ducis et interprétait ses autres productions d'inspiration shakespeariennes ainsi que sa création $^{34}$, mais pas son Hamlet. Aussi cette reprise était-elle très attendue. 
14 En 1787, Ducis, alors académicien de cinquante-quatre ans au faîte de la gloire, avait prédit à Talma une brillante carrière ${ }^{35}$. En 1803, les rôles s'étaient inversés. Agé de soixante-dix ans, ayant subi des aléas, Ducis acceptait volontiers que l'ancien élève passe maître. Talma avait modifié certains passages en se référant au texte original ${ }^{36}$ puisqu'il maîtrisait parfaitement l'anglais, ayant passé sa jeunesse à Londres où son père s'était installé3 ${ }^{37}$. Il y avait fréquenté assidument les théâtres et avait établi des liens avec les plus grands tragédiens de l'époque, comme John Philip Kemble ${ }^{38}$.

15 A cette reprise, de nombreux ex-détenus étaient venus honorer Labussière, dont Joséphine de Beauharnais, accompagnée du Premier Consul qui avait bénéficié de l'hospitalité de Talma ${ }^{39}$ lorsqu'il était arrivé à Paris démuni et sans appui. Talma avait traduit le soliloque « to be or not to be $»^{40}$. Cependant, même si son édition anglaise et Le Tourneur avaient réintroduit l'«Ombre" du Père d'Hamlet et le personnage de Fortimbras, ils n'apparaissent toujours pas à la scène, Talma restant fidèle à la version de Ducis. En revanche, la violence rétributive d'Hamlet et l'urne funéraire, furent deux moments qui contribuèrent à la gloire de Talma et restèrent à la postérité comme le prouve la peinture d'Anthelme Lagrenée présentée au Salon de 1810. 

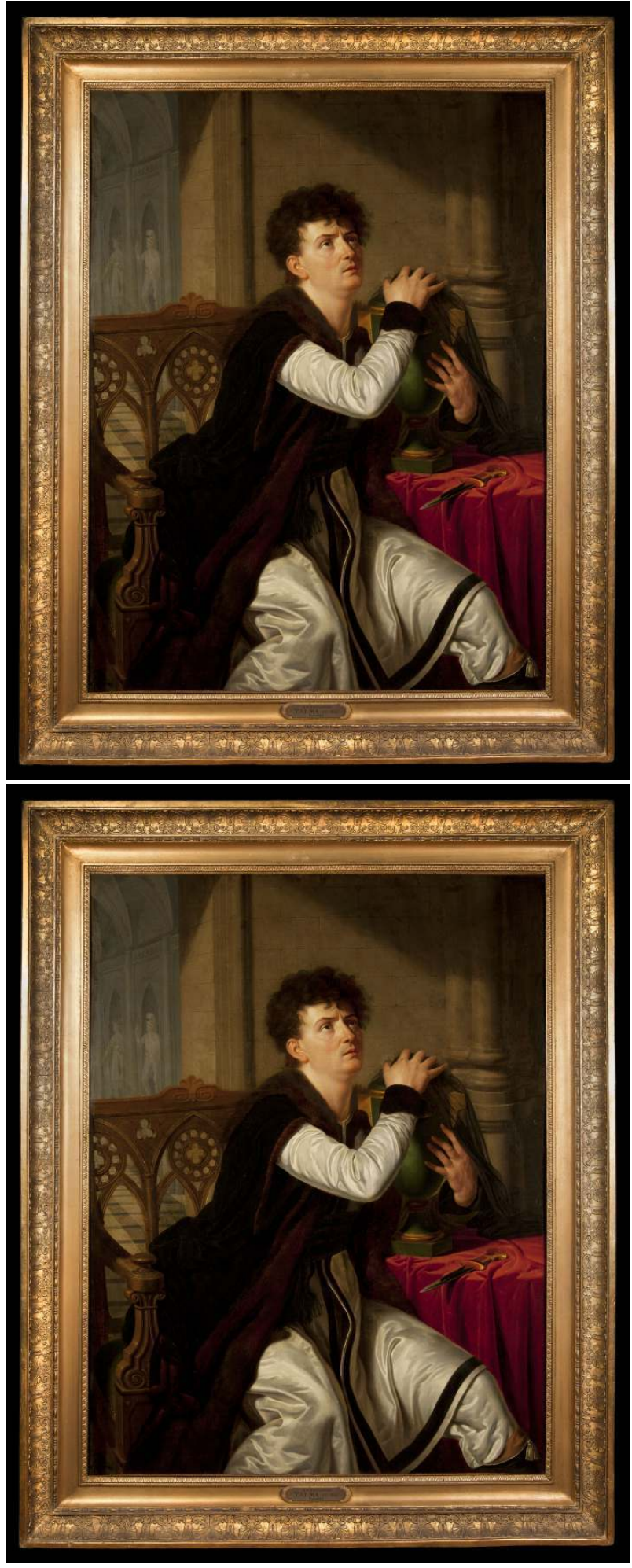

Illustration 2: Talma en Hamlet (Hamlet de Ducis) / Anthelme François Lagrenée, 1810, huile sur toile (I 0090)

(c)A. Dequier, coll. Comédie-Française 
La pièce fut constamment remaniée, toujours à cause de cet acte $\mathrm{V}$ qui ne satisfaisait pas Talma. Ducis, qui appelait Talma « mon Othello, mon Macbeth, mon Hamlet », se prêta au jeu avec enthousiasme durant les longues sessions de travail dans la maison de campagne de Talma, comme avant la reprise de 1805: "Je suis fort en état de travailler au cinquième acte de ma tragédie d'Hamlet. Tout a été fixé dans le travail que j'ai fait à Brunoy. [...] Je n'ai plus qu'à faire des vers, en conservant pourtant un certain nombre de ceux que j'ai déjà faits. Dès que Talma aura cet acte entre les mains, il jouera cette tragédie à Paris ${ }^{41} »$. Lorsque Ducis se sentait trop faible pour aller jusqu'à Brunoy, c'était seul, avec Népomucène Lemercier ${ }^{42}$ ou même Chateaubriand que Talma reprenait le texte.

17 Au cours de sa carrière Talma joua Hamlet soixante-quatorze fois au Théâtre-Français, faisant de ce rôle son sixième succès sur la première scène théâtrale ${ }^{43}$, sa dernière interprétation ayant eu lieu le 23 mai 1826, quelques mois avant sa mort (19 octobre 1826). De plus, Hamlet fut incontestablement le triomphe de son répertoire personnel lors de ses longues tournées privées. Lorsqu'il voyait le spectre, Talma, le poignard à la main, ne se livrait pas à une gestuelle braillarde comme Molé $^{44}$, mais restait là, immobile, faisant sentir l'effroi de cette apparition par la profondeur de son regard sombre (de myope) qui mesmérisait les spectateurs. À Arras, pendant la représentation du 31 juillet 1817, «[...] au moment où Hamlet lève son poignard sur sa mère, un officier qui se trouvait au parterre, saisi par l'horreur de la situation, poussa un cri et s'évanouit. Revenu à lui il demande avec angoisse: 'L'a-t-il tuée ?' » ${ }^{45}$. Cette réaction montre l'intensité du jeu scénique des acteurs; elle prouve aussi que l'officier ne connaissait ni la pièce ni l'ouvrage de Madame de Staël, De l'Allemagne, paru en France cette même année. Celle qui admirait tant l'acteur et qui avait finalement été autorisée à quitter son exil de Coppet pour assister à une représentation à Lyon en juin 1809, rendit compte de ce moment dans son chapitre sur « la déclamation»:

Les spectateurs ne voient pas l'ombre du père d'Hamlet sur la scène française, l'apparition se passe en entier dans la physionomie de Talma, et certes elle n'en est pas moins effrayante. Quand, au milieu d'un entretien calme et mélancolique, tout à coup il aperçoit le spectre, on suit tous ses mouvements dans les yeux qui le contemplent, et l'on ne peut douter de la présence du fantôme quand un tel regard l'atteste ${ }^{46}$.

18 La pièce de Ducis, qui appartenait au répertoire du Théâtre-Français, fut jouée avec des fortunes diverses et pour la dernière fois le dimanche 29 juin 1851, bien après la mort de Ducis en $1816^{47}$ et celle de Talma en 1826. Mais alors, une autre version de la pièce avait fait son chemin sur la scène théâtrale parisienne.

\section{Le drame d'Alexandre Dumas père (1802-1870) et Paul Meurice (1818-1905)}

\section{L'Époque romantique}

19 Le premier contact d'Alexandre Dumas avec Hamlet fut la version de Ducis qu'interprétaient des élèves du Conservatoire venus à Villers-Cotterêts, sa ville natale ${ }^{48}$. Puis, en 1827, comme tous les jeunes gens férus de nouveautés, dont Delacroix ${ }^{49}$, Dumas s'était précipité au Théâtre de l'Odéon pour admirer Charles Kemble et assister aux débuts aussi spectaculaires qu'éphémères d'Henrietta Smithson, l'égérie tragique d'Hector Berlioz. Comme il s'en est vanté ensuite dans ses Mémoires, il connaissait si bien la pièce qu'il n'avait pas jugé nécessaire de se procurer le livret bilingue qui se vendait au 
théâtre ${ }^{50}$. Il ajouta, cependant, que cette représentation l'avait bouleversé : «A partir de cette heure seulement, j'avais une idée du théâtre [...] C'était la première fois que je voyais au théâtre des passions réelles, animant des hommes et des femmes en chair et en os $^{51} »$.

\section{6 : Saint-Germain en Laye ; 1846-47 : Paris}

Dans l'engouement shakespearien de l'époque, Dumas y alla aussi de sa plume. Après une ébauche antérieure ${ }^{52}$, il écrivit, avec la participation de Paul Meurice, un texte complet qui fut joué en 1846 dans le petit théâtre de Saint-Germain-en-Laye ${ }^{53}$. Le succès fut tel que la pièce fut transférée à la saison suivante au Théâtre Historique à Paris ${ }^{54}$. Ce n'était plus une "tragédie », mais un «drame », en cinq actes, en vers. Au dénouement, suivant en cela Ducis, Hamlet était condamné à vivre, cependant, cet ordre lui venait du Spectre de son père, représenté physiquement sur scène $\mathrm{e}^{55}$. Théodore Massiac loua cette version qui rendait «les sublimes beautés de l'original. Plus d'édulcoration, plus d'atténuation, horreur, cruauté, démence : [les auteurs] avaient tout mis $»^{56}$. Théophile Gautier déplora l'absence de Fortimbras qui permettait une opposition entre l'homme de pensée et l'homme d'action ${ }^{57}$, mais il rendit hommage à l'interprète. Le rôle-titre était tenu par Philibert Rouvière (1809-1866), tout de noir vêtu, suivant la tradition scénique devenue canonique ${ }^{58}$. Sa frêle silhouette nerveuse, agitée de mouvements brusques et saccadés, véritable «tour de force qui fera date dans l'histoire du théatre" fit l'admiration de Charles Baudelaire qui vit en lui l'épitomé de «l'acteur romantique», par son interprétation et sa vie « agitée et tordue ${ }^{59}$. Rouvière aussi avait vu Hamlet à l'odéon en 1827 et avait calqué son jeu sur celui de Charles Kemble, l'éventail d'Ophélie à la main. Mais la tonalité de l'interprétation avait changé, comme en témoigne un jeu de scène mémorable, digne du grand guignol :

Et quand il tuait Polonius, il se précipitait comme un fou en criant : au rat, au rat! disparaissait derrière le rideau qui cachait le vieillard; on entendait le bruit d'une lourde chute, et Hamlet sortait lentement, à reculons, pour se retourner bientôt... on le voyait alors, tenant à la main sa petite épée de cour, qu'il secouait trois fois pour en faire tomber le sang de sa victime, en la regardant avec une fixité si effrayante, qu'un frisson de terreur glaçait toute la salle ${ }^{60}$.

21 Ce fut le rôle de sa vie, « son » rôle, et c'est ainsi qu'Edouard Manet l'a représenté en 1865, juste avant sa mort. Mais il s'identifia tant à Hamlet qu'il se fit vampiriser par le personnage, dans un accès extrême de romantisme.

\section{6 : Madame Judith Théâtre de la Gaité}

Une autre interprétation mérite d'être évoquée, même brièvement, celle de Madame Judith, au Théâtre de la Gaité en $1866^{61}$ sur un texte que Paul Meurice publia en son nom propre en 1864 dans lequel le personnage de Fortimbras avait été rétabli. S'inscrivant dans la tradition des Hamlet travestis qui firent fureur à la fin du XIXe siècle, Madame Judith, elle aussi, arborait l'éventail de la tradition anglaise.

Le rôle a été interprété sans discontinuer, mais connut un nouveau succès éclatant par Mounet-Sully. 


\section{Mounet-Sully (Jean-Sully Mounet, 27 février 1841-1er mars 1916)}

\section{6 : 28 septembre : Comédie-Française} se jouait au Théâtre de la Porte Saint-Martin dans une adaptation commandé à Lucien Cressinois et Charles Samson par Sarah Bernhardt qui interprétait Ophélie alors qu'elle était âgée de 42 ans, ce que les critiques n'ont pas manqué de lui faire remarquer. Edmond Got, qui allait être Polonius à la Comédie-Française, assista à la première, le 27 février ; son compte-rendu, peu favorable, rassura les Comédiens-Français ${ }^{62}$. Leur projet avait été initié bien avant par Emile Perrin, l'Administrateur de la Comédie-Française. En effet, en 1879, lors d'une tournée à Londres ${ }^{63}$, les Comédiens-Français avaient vu Henry Irving au Lyceum Theatre dans une interprétation nouvelle, leste et libéré des traditions, qui avait beaucoup de succès ${ }^{64}$. Emile Perrin engagea les préparatifs du spectacle courant 1885 , mais mourut quelques mois plus tard ${ }^{65}$. Son successeur, Jules Claretie, fut obligé de mener à terme ce projet ambitieux déjà bien avancé ${ }^{6}$.

Une édition de la pièce fut imprimée, avec les modifications apportées par Meurice (Dumas était mort en 1870) pour ce « Drame en cinq actes, en vers, composé de treize tableaux ${ }^{67} \%$. Lui-même suivait les répétitions, procédant le cas échéant à des modifications supplémentaires suggérées par les acteurs. La critique fut unanime pour louer cette interprétation qui durait quatre heures et trente minutes, un long entracte ponctuant chaque acte pour procéder aux changements de décors. C'était un spectacle grandiose, avec des décors impressionnants, des cortèges royaux à la nombreuse figuration, des passes d'armes, des airs nouveaux composés par Ambroise Thomas. Le style Renaissance avait été choisi par analogie avec la période de l'écriture de la pièce et non le temps de l'action. De somptueux costumes avaient été taillés dans de riches étoffes aux couleurs vives, ce qui produisait le plus bel effet, surtout dans les scènes de Cour ou durant les cortèges. Hamlet tranchait dans son vêtement de deuil, quoiqu'il fût richement ouvragé comme il sied à un Prince de rang. Parmi ces déploiements de fastes et de couleurs, l'interprétation de Mounet-Sully focalisa l'attention de la critique, Mallarmé, pourtant d'inspiration symboliste, se reconnut dans cette interprétation emprunte de romantisme ${ }^{68}$. Hamlet paraissait jeune (l'acteur avait pourtant quarante-cinq ans), naïf et doux ; il était tantôt pensif, en arrêt devant le médaillon de son père qu'il portait autour du cou ou dans les longues tirades dites de sa voix chaude au vaste registre, tantôt agile et souple lors de ses mouvements et déplacements esthétisés. Hamlet, qui se donna quarante-deux fois en 1886 et trente-quatre l'année suivante, devint l'événement de son temps ${ }^{69}$. 


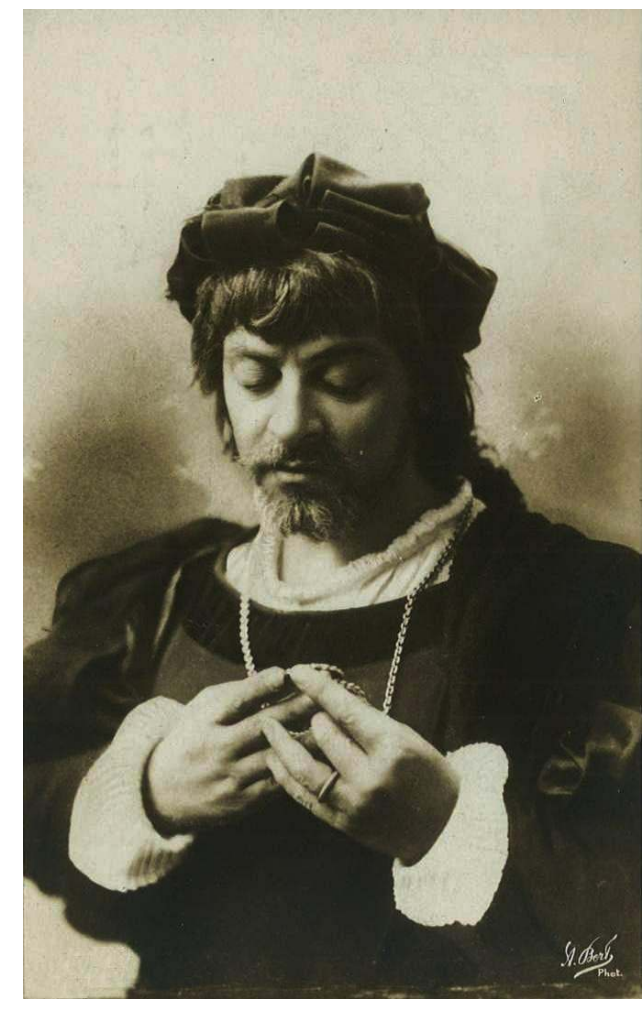

Illustration 3 : Mounet-Sully au médaillon, Cliché A. Bert

Collection Frédérick Sully, avec son aimable autorisation.

Le Spectre avait été rétabli dans le premier et le quatrième tableau. Il apparaissait à l'arrière-plan en pleine lumière argentée, à grand renfort d'effets spectaculaires ${ }^{70}$. A l'acte III, au 9è tableau, la didascalie indique que « le spectre du père d'Hamlet apparaît, visible pour Hamlet seul» (p. 93). C'était à l'acteur de faire sentir l'effroi dont il était saisi. Le regard de Mounet-Sully avait une telle intensité dramatique qu'il inspira de nombreux artistes, comme le portrait de Jean-Paul Laurens, présenté au Salon de $1888^{71}$. 


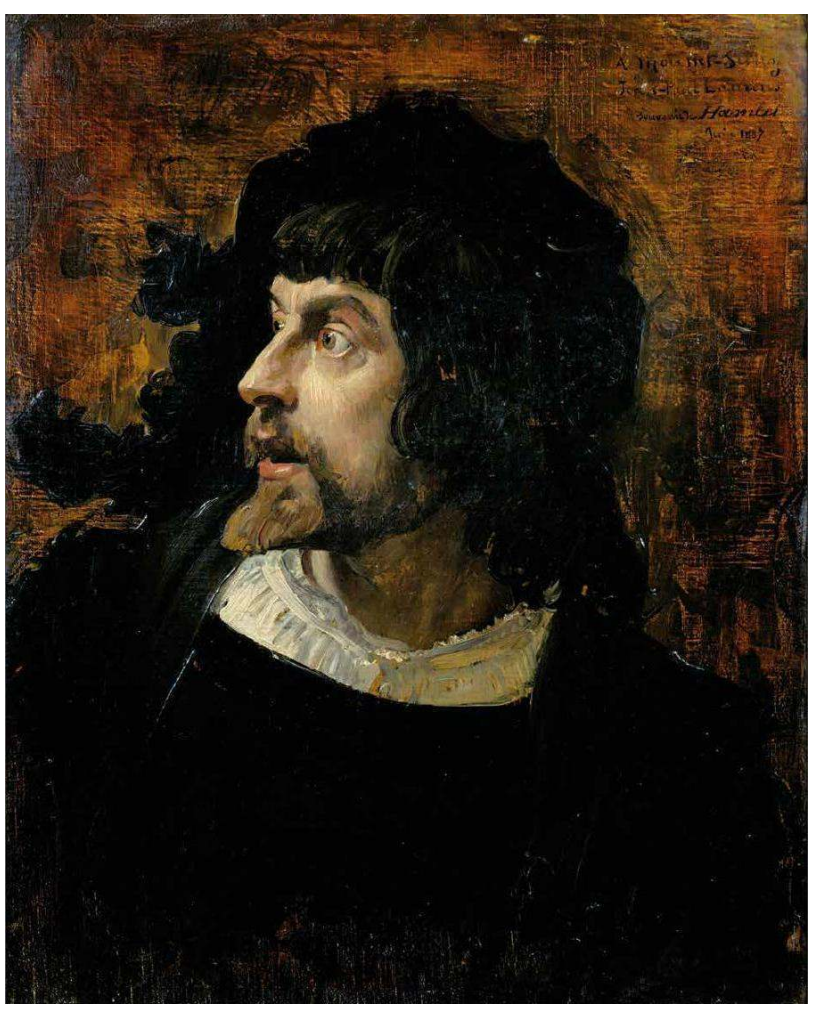

Illustration 4 : Peinture de Jean-Paul Laurens, 1887

Collection Frédérick Sully, avec son aimable autorisation.

Le 11e tableau, formant le début de l'acte $V$, représentait « un cimetière " (p. 113-124). Après un échange rapide avec les deux fossoyeurs, Hamlet s'emparait du crâne de Yorick: ce moment très émouvant sur le devant de la scène a été maintes fois reproduit. Hamlet et Horatio se dissimulaient ensuite à jardin pour laisser passer le cortège funèbre qui partait du fond de scène pour remonter majestueusement vers la cour, traversant tout l'espace scénique.

28 Le Théâtre dans le Théâtre, placé à l'Acte III, scène 2 (7e tableau), produisait un effet scénique si surprenant par son imposante mise en scène que ce moment a frappé jusqu'à Jean Cocteau qui, en rendant hommage à Mounet-Sully en 1966, évoqua «le prince Hamlet rampant aux pieds de sa mère un éventail à la main ${ }^{72} »$. Mounet-Sully avait emprunté ce jeu de scène à la tradition anglaise dont Delacroix rendit compte lors de son séjour à Londres $1825^{73}$ et qu'il a immortalisé après le passage de Charles Kemble à l'Odéon en 1827, ainsi qu'Achille Devéria avec son dessin ${ }^{74}$. Philibert Rouvière, lui aussi, reprit ce moment au Théâtre Historique en 1847. En 1886, ce fut au tour de Mounet-Sully d'interpréter ce passage canonique dont la tradition scénique avait fixé le jeu, et ce, plus de soixante ans après Charles Kemble. Néanmoins, Mounet-Sully avait complètement réinventé ce moment dramatique par cette façon qu'il avait d'agiter frénétiquement l'éventail d'Ophélie à la tête de Gertrude. C'était même devenu l'un des emblèmes de l'époque et de son jeu. 


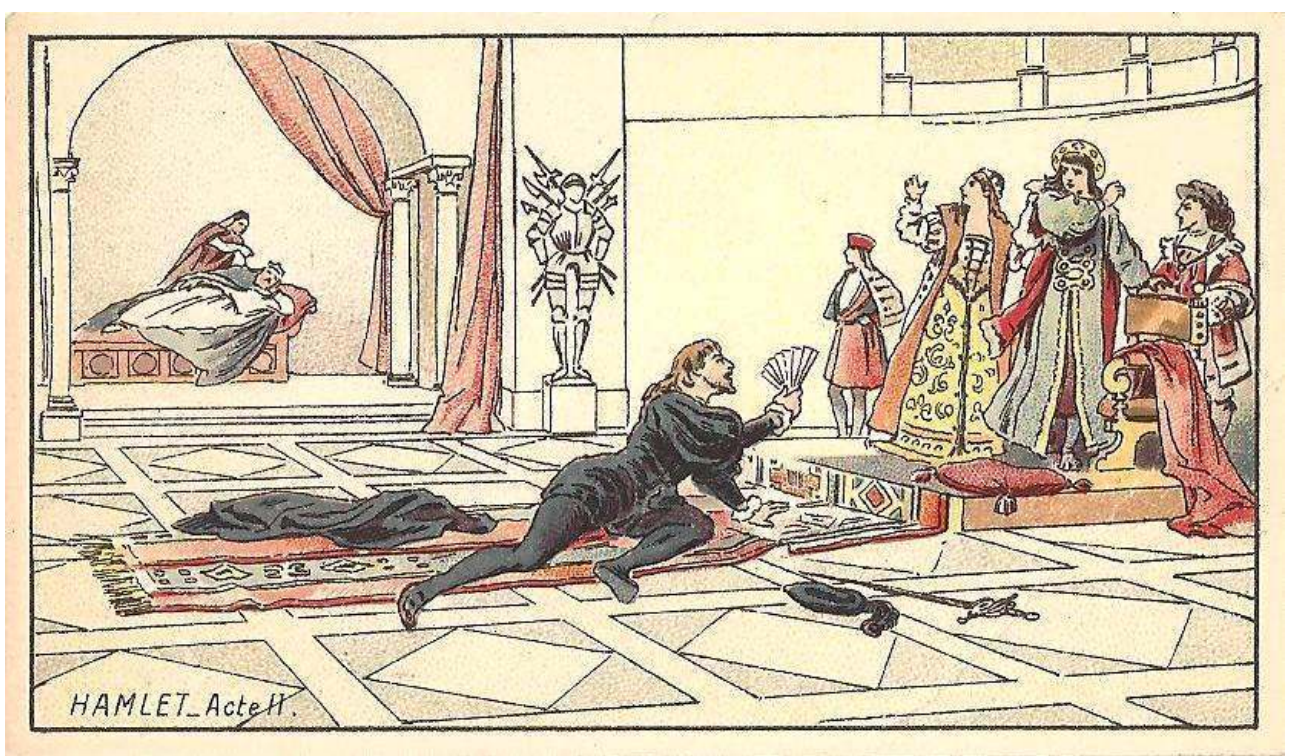

Illustration 5 : Acte II, Chromo, Publicité « Au Tisserand »

Collection Frédérick Sully, avec son aimable autorisation.

\section{4 : tournée en Amérique}

De mars à fin mai 1894, Mounet-Sully partit en tournée en Amérique avec une troupe de la Comédie-Française. Les représentations d'Hamlet y furent données la troisième semaine du séjour, avec une édition bilingue imprimée pour l'occasion ${ }^{75}$. La « ressemblance immortelle » entre le personnage et l'interprète que Mallarmé avait tant admirée en Mounet-Sully était, à son tout, balayée, par une nouvelle manière de jouer. Si le XVIIIe siècle hésitait à monter Hamlet, le XIXe siècle en fit la pièce emblématique par excellence de son époque, dans des interprétations exclusives qui se sont succédées, centrées sur le personnage éponyme à travers des artistes d'exception qui portaient, à eux seuls, le message de Shakespeare. 


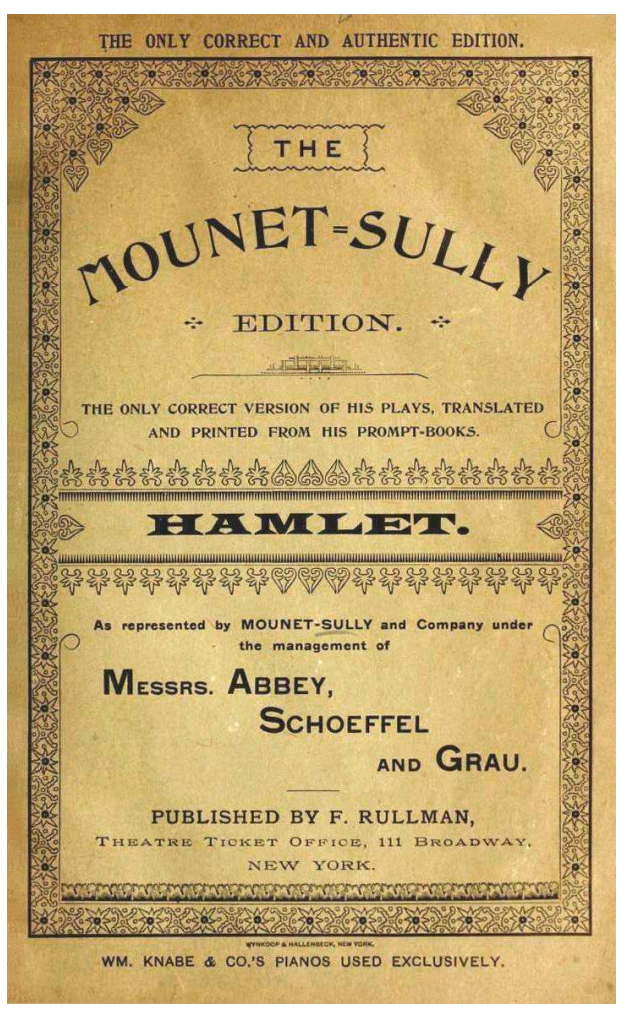

Illustration 6 : Edition bilingue de Hamlet, Couverture, New York, F. Rullman, 1894

Collection Frédérick Sully, avec son aimable autorisation.

Alors qu'il avait été acclamé comme un « dieu » à Bucarest, il fut parfois obligé de jouer devant des salles quasiment vides. Pourtant, les critiques furent très favorables, mais peut-être pas les circonstances: Henry Irving venait juste de terminer une tournée triomphante dans la langue originale et selon une gestuelle beaucoup plus sobre. Il n'y avait pas ces décors somptueux qui faisaient rêver les Parisiens, la distribution laissait à désirer. Et de plus, les Américains s'intéressaient peu au drame. Avant de quitter NewYork, Mounet-Sully constata avec une certaine amertume qu'aux abords du théâtre, presque vide lors de ses prestations, se pressait alors une foule pour voir « une féérie compliquée d'exhibitions chorégraphiques et zoologiques ${ }^{76}$ ».

\section{6 : reprise}

31 Mounet-Sully retrouva la Comédie-Française et ses spectateurs pour une reprise en 1896. Les répétitions reprirent car il s'agissait d'une véritable re-création avec une distribution différente en présence de Paul Meurice qui avait encore modifié son texte. Des suggestions de Mounet-Sully avaient été prises en compte : le personnage de Fortimbras fut restauré, les tableaux avaient été réduits à neuf, supprimant ainsi plusieurs entractes et permettant un rythme plus fluide en évitant de briser l'illusion théâtrale.

\section{1 : Dernière}

En 1910, ce spectacle fut aussi donné en plein air au Théâtre antique d'Orange, et l'année suivante, Mounet-Sully incarna Hamlet pour la dernière fois. Il avait soixante-dix ans, la chevelure et la barbe complètement blanchies; Ophélie était jouée par sa jeune 
compagne, Jeanne Rémy. Ce fut un triomphe. Cela faisait exactement vingt-cinq ans qu'il interprétait ce rôle qui fut l'un de ses grands succès incontestés.

\section{Conclusion}

Les débuts d'Hamlet sur la scène française furent arrachés de haute lutte par Ducis. Et pourtant, cette pièce, tragédie, puis drame, devint emblématique de la scène française au XIXe siècle, grâce à deux interprètes majeurs qui ont incarné l'esprit de leur temps, l'Empire puis la Restauration pour Talma, le post-Romantisme pour Mounet-Sully. Des prédécesseurs illustres, Molé puis Rouvière, avaient créés le rôle, mais, par leur interprétation et leur longévité à la scène, Talma et Mounet-Sully les ont éclipsés par leur art qui, d'abord, à l'encontre des pratiques existantes, a marqué de leur sceau leur époque. Tous deux ont collaboré longuement avec l'auteur, modulant le texte de leur empreinte renouvelée.

Tout passe, sauf Hamlet. Tandis que Mounet-Sully jouait à la Comédie-Française, à l'Exposition Universelle de 1900, Sarah Bernhardt en rôle travesti, interprétant la scène du duel sur pellicule animée, électrisa la Belle Epoque. La "ressemblance immortelle» entre le personnage et l'interprète que Mallarmé avait tant admirée en Mounet-Sully était, à son tout, balayée, par une nouvelle manière de jouer. Si le XVIIIe siècle hésitait à monter Hamlet, le XIXe siècle en fit la pièce emblématique par excellence de son époque, dans des interprétations exclusives qui se sont succédées, centrées sur le personnage éponyme à travers des artistes d'exception qui portaient, à eux seuls, le message de Shakespeare.

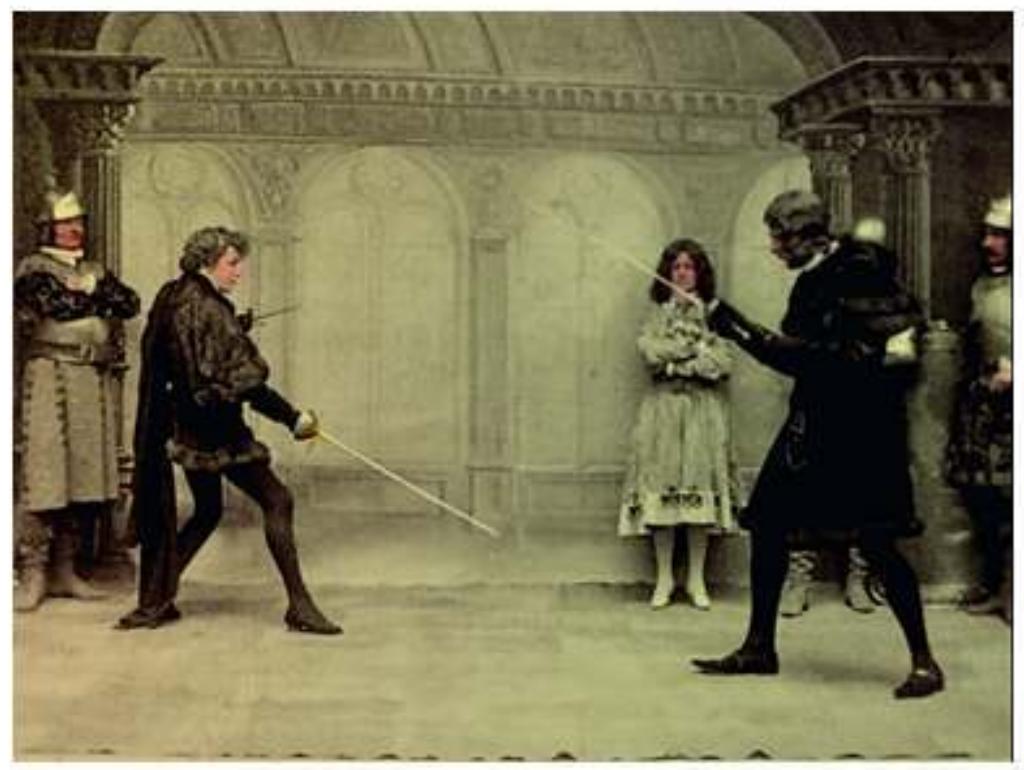

Sarah Bernhardt et Pierre Magnier, 1900 
Illustration 7 : capture d'écran. Vue colorisée « Photo-Cinéma-Théâtre » selon le procédé GratiouletLioret, 20 mai 1899. Sarah Bernhardt (Hamlet), Philippe Garnier OU Pierre Magnier (1869-1959) (Laërte). Théâtre de la Porte Saint-Martin, traduction Eugène Morand et Marcel Schwob (Paris, Charpentier \& Fasquelle, 1900).

Vidéo disponible sur Youtube : film de 3 minutes N/B « Duel d'Hamlet » de Clément Maurice (1853-1933), https://www.youtube.com/watch?v=aXh9IbESHAO

\section{NOTES}

1. Le Jubilé eut lieu du 6 au 8 septembre 1769. Michael Dobson, The Making of the National Poet: Shakespeare, Adaptation and Authorship, 1660-1769, Oxford, Clarendon Press, 1992.

2. Jean-François Ducis (1733-1816), Hamlet : tragédie imitée de l'anglois, Paris, Gogué, 1770.

3. Pierre-Antoine de La Place (1707-1793), Théâtre Anglois, 8 vol., in-12, Londres-Paris, Vve Pissot, 1746-1749. Hamlet est la deuxième pièce du vol. 2, p. 312-355. La Place a tantôt traduit, tantôt résumé, tantôt commenté certains passages qu'il s'était senti obligé de reproduire tant ils étaient connus, sauf le soliloque « to be or not to be » que Voltaire avait traduit dans sa lettre XVIII.

4. Lillian Cobb, Pierre-Antoine de La Place. Sa vie et son oeuvre, 1707-1793, Paris, E. de Broccard, 1928 et le site www.guyane.com

5. Alexander Pope (1688-1744), The Works of Shakespeare. In six volumes. Collated and corrected by the former editions. London, Jacob Tonson, 1725. Hamlet figure dans le volume 6, p. 343-472. La deuxième édition, en dix volumes, a paru aussi chez Tonson en 1728.

6. John Golder, Shakespeare for the Age of Reason, Oxford, The Voltaire Foundation, 1992, p. 7 et p. 14, note 5. Bien qu'appartenant à deux générations différentes, les deux hommes se connaissaient et se retrouveront en 1777 parmi les invités de Beaumarchais, pour former le «Bureau de législation dramatique ». Voir Alan Kors, D’Holbach's Coterie. An Enlightenment in Paris, Princeton, Princeton University Press, 1976.

7. Séjours de Garrick à Paris : 1751 (10 jours), 1763 (deux semaines), 1765 (plus de six mois, convalescence après son périple en Italie et en Allemagne). F.A. Hedgcock, David Garrick et ses amis français, Paris, Hachette, 1911.

8. Le grand-père de David Garrick, David de la Garrigue/ou de la Garrique avait quitté Bordeaux à la Révocation de l'Edit de Nantes en 1685 et anglicisé son nom en Garric lors de son installation en Angleterre. Voir Peter Holland (éd.), Garrick, Kemble, Siddons, Kean: Great Shakespeareans, Londres, Bloomsbury, 2010.

9. Lettre du 14 avril 1769, citée par John Golder, op. cit., p. 20.

10. Amélise, jouée le 9 janvier 1768 avait connu quelque succès. Voir Sylvie Chevalley, «Ducis, Shakespeare et les Comédiens-Français», $1^{\text {ère }}$ partie, De Hamlet (1769) à Roméo et Juliette (1772), in Revue d'Histoire du Théâtre, 1964 (4), 237-350, p. 329-330.

11. Le 19 août 1768, le quorum n'étant pas atteint, le Comité avait reporté la deuxième lecture au lendemain. Ce jour-là, le manuscrit n'avait été accepté que par huit des vingt-trois votants, douze voix avaient demandé des modifications. Le 5 mai 1769, le Comité ne put encore siéger, mais, lors de la réunion du 17 juillet, le Comité accepta la pièce (Sylvie Chevalley, ibidem).

12. Henri-Louis Caïn, dit Lekain (1729-1778), débuta au Théâtre-Français en 1750. Apprécié pour sa diction, il se fit l'interprète privilégié du répertoire classique et de Voltaire. Il mourut juste 
avant le philosophe. "M. Ducis, dit Campenon, attribua ce refus à l'influence de Voltaire » in Sylvie Chevalley, op. cit., p. 331.

13. Sylvie Chevalley, ibidem, cite une lettre de Ducis à son ami d'enfance Vauchelle: "Toute la compagnie a été transportée; il n'y a eu qu'une voix pour la recevoir. On a trouvé mes corrections admirables, et j'ai reçu des compliments de tous côtés. Il y a mieux, j'ai distribué mes rôles hier, tant la Comédie est empressée de me jouer et espère de l'ouvrage. Molé [1734-1802] m'a garanti le succès ; il est charmé du rôle d'Hamlet ».

14. Site La Grange : www.comedie-francaise.fr.

15. John Golder, op. cit., précise «Lancaster [I, p. 825] records 896 paying spectators, but this figure does not include persons on the theatre's 'free list' or persons using the annually hired petites loges », p. 18, note 28.

16. Sylvie Chevalley, op. cit: Samedi 30 septembre : 3.033,10 f ; mercredi 4 octobre : 2.406 ; Samedi 7 octobre : 2.055 ; mercredi 11 octobre : 2.098 ; samedi 14 octobre : 1.627.

17. Louis Petit de Bachaumont (1690-1771), auteur supposé des Mémoires Secrets, 3 octobre 1679. Cité par John Golder, op. cit. p. 44.

18. Denis Diderot (1713-1784), Euvres Complètes, vol. VIII, p. 471-476, cité par John Golder, op. cit., p. 47 .

19. Lettre du 14 avril 1769, citée par John Golder, op. cit., p. 20.

20. Sémiramis, tragédie de Voltaire (1694-1778), créée le 29 août 1748.

21. Charles Collé (1709-1783) est l'auteur de La Partie de Chasse d'Henri IV (1762) dont l'air «Vive Henri IV » devint un hymne royaliste durant le règne de Louis XVI. John Golder, op. cit.: “M. Ducis [...] est né sans génie, sans talent et sans invention. Non seulement il n'a rien imaginé lui-même dans le sujet d'Hamlet, mais il a même gâté cette tragédie brute", p. 45.

22. L'Année Littéraire, 1770, t. II, p. 293-319, cité par Sylvie Chevalley, op. cit., p. 333.

23. John Golder, op. cit., p. 46.

24. Les manuscrits sont consultables à la Bibliothèque-Musée de la Comédie-Française.

25. Marion Monaco indique que Molé remporta un tel succès qu'il joua le répertoire tragique après cette interprétation. Shakespeare on the French Stage in the 18th Century, Paris, Didier, 1974, p. 77.

26. Madame du Deffand [1697-1780] in Journal de Paris, 17 Germinal An XI/7 avril 1703, cité par Sylvie Chevalley, op. cit., p. 337.

27. Ducis, lettres à Garrick du 14 avril 1768 citée par Sylvie Chevalley, op. cit. p. 332 et note 13 p. 332 : L'impropriété de l'entretien d'Hamlet avec les comédiens sera soulignée par L'Année littéraire, 1779, VI, 317 : « Un prince qui instruit un comédien sur son art joue un personnage peu convenable à sa dignité ».

28. Diderot, Euvres Complètes, VIII, p. 475, cité par Marion Monaco, op. cit., p. 71

29. Cité par John Golder, op. cit. Golder note néanmoins la voix discordante de Charles Collé, p. 46. 30. Jean-François Ducis, Hamlet : tragédie imitée de l'anglois, op. cit. p. 68.

31. Mara Fazio, François-Joseph Talma [1766-1826]. Le Théâtre et l'Histoire, de la Révolution à la Restauration, Paris, CNRS-Editions, 2011 (1999 pour l'édition italienne) : "l'ancienne salle de l'Opéra, louée pour l'occasion par le Théâtre Favart ", p. 124.

32. Pamela ou la vertu récompensée de François de Neufchâteau (1750-1828), version française de la pièce de Goldoni, Pamela nubile, imitée du roman épistolaire de Richardson. Dans la nuit du 3 au 4 septembre 1793 les Comédiens-Français (sauf Molé) et Neufchâteau furent emprisonnés à la prison des Madelonnettes (Mara Fazio, op. cit., p. 73-74). Voir aussi Charles-Guillaume Etienne (1777-1845) et Alphonse Martainville (1776-1830), Histoire du Théâtre Français depuis le commencement de la Révolution jusqu'à la réunion générale, 4 vol., Paris, chez Barba, An X, 1802.

33. Louise Contat avait envoyé une lettre aux journaux le 25 mars 1795 disculpant Talma. Herbert Collins, Talma, a Biography of an Actor, Londres, Faber \& Faber, 1964, p. 94-95. 
34. Du répertoire shakespearien de Ducis, Talma avait créé Jean Sans-Terre qui devait commencer le 21 juin 1791, mais fut reportée au 28 juin, à cause de la fuite et capture de la famille royale à Varennes et ne fut joué que sept fois, sans grand succès. Othello suivit en novembre 1792. Talma avait repris le rôle-titre de Macbeth en janvier 1792 et celui du roi Léar (créé par Brisard) en juin 1792. Il avait eu beaucoup de succès dans le rôle d'Abufar, la pièce de Ducis, Abufar, ou la famille arabe, en avril 1795.

35. Le 21 novembre 1787, à 24 ans, juste sorti de la première promotion du Conservatoire, Talma débuta dans le rôle de Seïde dans Mahomet, la tragédie de Voltaire.

36. Comme il l'avait fait pour Othello en 1792, voir Maria Fazio, op. cit., p. 124. Talma avait les œuvres complètes de Shakespeare en trois volumes du Reverend Samuel Ayscough publiées en 1790 et la traduction de Pierre Le Tourneur (1737-1788), Le Théâtre de Shakespeare, dédié au roi, 20 vol. Paris, Vve Duchesne, 1776-1783. Hamlet Prince de Dannemarck est dans le volume 5 (après Le roi Léar).

37. Son père officiait comme dentiste.

38. À la soirée d'adieu de John Philip Kemble (1757-1823) où il interprétait son rôle emblématique de Coriolan, le 23 juin 1817, Talma fut applaudi pour avoir répondu à un toast dans un anglais irréprochable.

39. Et de Julie Carreau, la femme de Talma, dont l'hôtel particulier se trouvait rue Chantereine, dans le quartier de la Nouvelle Athènes à Paris.

40. Témoignage de Friedrich von Schlegel (1772-1829), le frère du traducteur de Shakespeare, qui résida à Paris de Juillet 1802 à avril 1804. Mara Fazio, op. cit., p. 124.

41. Lettre de Ducis à son ami Soldini de Versailles (17 Vendémiaire XIV/9 Octobre 1805) citée par John Golder, op. cit., p. 57.

42. « En 1810 encore, c'est la collaboration Ducis-Talma-Lemercier qui fixe les textes définitifs d' Hamlet et de Macbeth » Sylvie Chevalley, op. cit., p. 335.

43. Sylvie Chevalley, op. cit., "Appendice ", p. 347. Napoléon n'étant pas en phase avec ce héros enclin à la méditation et à la procrastination, il exigeait le répertoire martial de Corneille ou de Racine.

44. Talma fut apprécié pour sa voix feutrée, mais critiqué par l'abbé Geoffroy [1743-1814] pour son manque de possibilités vocales par comparaison avec Lekain et Pierre Lafon.

45. François et Madeleine Ambrière, Talma ou l'histoire au théâtre, Paris, Fallois, 2007, p. 561. En 1817, Talma ne fut pas autorisé par le duc de Duras à passer par Bruxelles sur le chemin du retour car les réfugiés libéraux étaient nombreux (dont Arnault et David), il continua sa tournée, par Mons, puis se rendit à Poix, berceau de sa famille paternelle, Douai, et Arras.

46. Germaine de Staël-Holstein (1766-1817), exilée à Coppet (Suisse) dans le domaine familial, fut autorisée à rentrer sur le territoire français avec un passeport spécial pour voir Talma. Son essai, De L'Allemagne, mis au pilori en France en 1810 fut publié à Londres en 1813 et en France en 1817. Paris, GF-Flammarion, vol. 2, p. 37.

47. Ducis est né et mort à Versailles (23 août 1733-31 mars 1816). Sylvie Chevalley, op. cit. «La nouvelle de sa mort fut portée au Registre des représentations journalières avec le commentaire : Rien ne peint mieux cet auteur célèbre, dont la longue carrière a été si honorable, que ce vers de M. Andrieux : “Accord d'un beau talent et d'un beau caractère », p. 328.

48. Jean Jacquot, « Mourir ! Dormir !... Rêver peut-être ? Hamlet de Dumas-Meurice de Rouvière à Mounet-Sully ", Revue d'Histoire du Théâtre, 1964, n4, p. 407-445. (Mes Mémoires, éd. P. Josserand, t. 1, 1954, p. 418-419 référencées par Jacquot note 1, p.407), Jacquot suppose que cette représentation eu lieu avant 1822, date à laquelle Dumas quitta Villers-Cotterêts.

49. Voir la lettre que Delacroix envoie à son ami Charles Soulier rendant compte de la représentation, Michèle Hannoosh, Eugène Delacroix - Journal, 2 vol., Paris, José Corti, 2009.

50. "Je savais si bien mon Hamlet que je n'avais pas eu besoin d'acheter le libretto ", t. 2, p. 419, cité par Jean Jacquot, op. cit., ibidem. 
51. Jean Jacquot, ibidem.

52. Dumas avait composé un premier jet de Hamlet en 1840, puis une version de Macbeth en 1842, refusée par le Comité de Lecture de la Comédie-Française et resté inédite.

53. Dumas, habitant alors à Saint-Germain-en-Laye, loua le petit théâtre de la ville pour une douzaine de représentations. Jean Jacquot, op. cit., p. 408.

54. Le théâtre, qui devait s'appeler Théâtre Montpensier, construit en 1846, fit faillite en 1850 et fut repris jusqu'en 1863 sous l'appellation Théâtre du Boulevard du Temple.

55. « Tu vivras ! ». L'Ombre distribue « prie et meurs! » à Laërte, « espère et meurs! » à Gertrude et « désespère et meurs! » au Roi, prouvant que Dumas connaissait aussi son Richard III, voir Jean Jacquot, op. cit., p. 417.

56. Théodore Massiac, (1851-1916), « Nos Hamlet », p. 389, non identifié, Dossier « Hamlet » de la Comédie-Française.

57. Théophile Gautier (1811-1872) cité par Jean Jacquot, op. cit., p. 417.

58. Le deuil se porte en noir à présent dans le monde occidental, mais cette pratique n'est pas attestée au Danemark à l'époque de l'action.

59. Charles Baudelaire, Ch. XI "L'acteur romantique », in L'art Romantique, Paris, Calmann-Lévy, 1869.

60. Théodore Massiac, op. cit.

61. Madame Judith (1827-1912) fut sociétaire de la Comédie-Française de 1852 à 1866.

62. Cité par Anne Penesco, Mounet-Sully, l'homme aux cent cœurs d'homme, Paris, Cerf, 2005: «La pièce, montée - décors et costumes- en style du XIIIe siècle, à ce que le directeur a nettement affirmé, a eu un succès certain de première, surtout grâce à la scène des fleurs de Mlle Sarah $B$, qui y a été remarquable. Notre ancien pensionnaire, M. [Philippe] Garnier, est un Hamlet un peu troisième rôle, mais avec la ligne générale du personnage, a eu deux ou trois coups de force applaudis. Le reste a été suffisant, voilà tout. PS : Et je crois nous sentir ici, dès à présent, beaucoup plus solides sous presque tous les rapports ", p. 219-220.

63. Anne Penesco : «Représentations londoniennes du 31 mai au 12 juillet 1879. Autour de Got remplaçant Perrin retenu à Paris par la santé de sa femme (qui meurt le 2 juin) : Mounet-Sully, Sarah Bernhardt, les Coquelin aîné et cadet, Delaunay, Worms, Maubant, Jenny Thénard. Jean Aicard, François Coppé, Francisque Sarcey sont aussi du voyage », p. 166-167.

64. Emile Perrin retourna à Londres en 1885 revoir Hamlet. Journal Parlementaire, 7 mai 1885 (Archives Frédérick Sully).

65. Emile Perrin meurt le 20 octobre 1885. Jules Claretie lui succède et restera en poste jusqu'au 23 décembre 1913. Anne Penesco, op. cit., p. 212.

66. Jules Claretie : rapports de l'Administrateur. Dossier Comédie-Française.

67. Alexandre Dumas-Paul Meurice, Hamlet Prince de Danemark, Paris, Calmann Levy, 1886.

68. Stéphane Mallarmé, "Notes sur le Théâtre », in Revue Indépendante, tome 1, Nov-Déc. 1886, p. 25-29.

69. Voir le tableau de Joannides, Dossier Comédie-Française.

70. Dans son rapport de "Semainier", cependant, Mounet-Sully avait noté la difficulté de synchroniser les variations lumineuses à ces moments cruciaux : il se plaignait du manque de coordination des machinistes et des gaziers. De plus, à l'affût de nouveautés, il induisit que l'éclairage au gaz était bien moins performant que l'électricité dont la salle de l'opéra venait juste de s'équiper. « Rapport du Semainier », Dossier Comédie-Française: les acteurs devaient prendre en charge ce rapport hebdomadaire à tour de rôle, notant surtout les problèmes rencontrés. Malgré l'importance de son rôle, Mounet-Sully s'est acquitté de ce surcroît de travail avec une grande conscience.

71. Tableau de Georges Clairin - ami intime d'Henri Regnault et portraitiste préféré de Sarah: Claudius en prière, Hamlet remet son épée au fourreau. On compare aussi Mounet-Sully avec le 
Hamlet de Delacroix « avec quelque chose de plus puissant encore », Henri de La Pommeraye (Les Premiers Illustrés, t. VI, Paris, Alphonse Piaget, 1887).

72. Jean Cocteau (1889-1955), «Portrait de Mounet-Sully», 6 juin 1966. Dossier Mounet Sully, Comédie-Française.

73. Arlette Sérullaz et Yves Bonnefoy, Delacroix \& Hamlet, Paris, Editions de la Réunion des Musées Nationaux, 1991, «Acte III, scène II, Hamlet fait jouer aux comédiens la scène de l'empoisonnement de son père », p. 34.

74. Achille Devéria (1800-1857) et Louis BOULANGER (1806-1867), Souvenirs du Théâtre Anglais à Paris, dessinés par MM. Devéria et Boulanger, texte M. Moreau, Paris, Henri Gaugain, Lambert et Compagnie, 1827, accessible en ligne sur http:/gallica.bnf.fr/ark:/12148/bpt6k62865096/ f8.item.r=souvenirs\%20du\%20theatre\%20anglais\%20a\%20paris.zoom, consulté le 20 décembre 2016.

75. Cependant, les deux versions n'offrent pas le même dénouement. (Archives Frédérick Sully). 76. Lettre/Journal à sa femme, Georgette Barbot. Dossier Mounet Sully, Comédie-Française.

\section{RÉSUMÉS}

Lorsqu'en 1803, François-Joseph Talma (1766-1826) reprend le rôle d'Hamlet que François Molé avait créé en 1769, il est le Comédien-Français préféré de Napoléon et entre ensuite dans une longue collaboration fructueuse avec Jean-François Ducis (1733-1816), assurant le succès du dramaturge autant que le sien propre que Madame de Staël a si bien décrit dans son ouvrage De l'Allemagne (1817). À l'époque romantique, Philibert Rouvière (1809-1866) s'empare du rôle dans une interprétation grandguignolesque encensée par Baudelaire. À l'autre extrémité du siècle, en 1886, également dans l'adaptation d'Alexandre Dumas père (1802-1870) en collaboration avec Paul Meurice (1818-1905), le personnage devient «le sceau d'une époque », le grand ComédienFrançais Mounet-Sully (1841-1916) marquera son temps par son lyrisme et «l'exorcisme d'un geste ", faisant du héros l'emblème fin-de-siècle que Stéphane Mallarmé qualifia de Hamlétisme. Mounet-Sully interpréta Hamlet sur la scène française et européenne pendant près de vingt-cinq ans, et ira plus loin encore que Talma, jusqu'en Russie et même aux Etats-Unis. En cette année 1886, la concurrence est grande puisque Sarah Bernhardt interprète Ophélie sur une autre scène parisienne, puis, en 1900, se travestit en Prince de Danemark avec tant de succès qu'elle restera gravée sur pellicule colorisée animée dans la scène du duel lors de l'Exposition Universelle de 1900.

When François-Joseph Talma (1766-1826) played Hamlet in 1803, a part that François Molé had premiered in 1769, he was Napoléon's favourite actor and was embarking on a long, fruitful cooperation with Jean-François Ducis (1733-1816), granting the success of the playwright as well as his own that Madame de Staël described so well in her essay De L'Allemagne (published in France in 1817). During the Romantic period Philibert Rouvière (1809-1866) turned the part into a horror melodrama which was highly praised by Baudelaire. At the other end of the century, in 1886, again in the adaptation of Alexandre Dumas (1802-1870) and Paul Meurice (1818-1905), Hamlet became the emblem of the time in the revival given by the great Comédie-Française actor, Mounet-Sully (1841-1916). He left his mark on his time thanks to his lyricism and refined gestures, showing the hero as the epitome of the turn of the century, praised by Mallarmé who coined the word Hamletism. Mounet-Sully played Hamlet on the French and European stage for 
over twenty-five years, and even travelled farther than Talma, as far as Russia and America. In 1886 competition was fierce on the Parisian stage as Sarah Bernhardt performed Ophelia; and then, in another production, played Hamlet with such gusto that her Duel against Laertes was shot on a colourized film and shown as an animated sequence at the 1900 World Exhibition in Paris.

INDEX

Mots-clés : Adaptation, Hamlet, interprétation, XIXe siècle français, Ducis Jean-François, Dumas Alexandre, Meurice Paul, Mounet-Sully, Talma François-Joseph, Shakespeare William

Keywords : Adaptation, Shakespeare William, Hamlet, performance, Mounet-Sully, Ducis JeanFrançois, Dumas Alexandre, Nineteenth Century in France, Meurice Paul, Talma François-Joseph

\section{AUTEUR}

\section{ISABELLE SCHWARTZ-GASTINE}

Université de Caen Normandie \& THALIM, CNRS Paris 